\title{
Next generation of Radiation Tolerant Single-Mode Optical Links for Accelerator Instrumentation
}

\author{
C. Scarcella ${ }^{1}$, M. Barros Marin, S. Détraz, R. Jones, A. Kraxner, L. Olantera, \\ C. Sigaud, C. Soós, C. Stile, J. Troska and F. Vasey \\ CERN \\ Esplanade des Particules 1, 1211 Geneva, Switzerland \\ E-mail: carmelo.scarcella@cern.ch
}

\begin{abstract}
Long-reach data transmission is an enabling technology for Accelerator Instrumentation at CERN. We present the development of next generation radiation-hard single-mode optical links. This new design aims to support the increasing data volume produced by the beam sensing electronics deployed along the accelerators. We present the design of the new optical data link and its characterization in terms of functional performance and radiation tolerance.
\end{abstract}

\section{${ }^{1}$ Speaker}




\section{Introduction}

The proton beam in the Super Proton Synchrotron (SPS) and Large Hadron Collider (LHC) is monitored at regular distance intervals by dedicated measurement systems, Figure 1. The acquired data is used in the feedback loop controlling the beam. Radiation-hard optical links, with a reach of up to $3 \mathrm{~km}$, are necessary to connect the beam sensing electronics with the radiationfree areas where the data are processed.

During Long Shutdown LS3 and LS4, part of the beam monitoring instrumentation will be upgraded. In order to achieve higher measurement precision, digital electronics will replace the current instrumentation based on analog circuits. The high volume of digital data foreseen for the High Luminosity LHC, and the necessity to monitor the beam bunch by bunch requires deployment of a new generation of single-mode radiation tolerant high-bandwidth optical links.

The Versatile Link project [1] developed a custom rad-hard transceiver (VTRx) targeting the upgrades of the LHC Experiments during Long Shutdown LS2. The VTRx is based on radiation tolerant laser driver and amplifier ASICs (GBLD [2] and GBTIA [3]), and commercial laser diodes and photodiodes which were validated for radiation tolerance. It is a bidirectional transceiver operating at $4.8 \mathrm{~Gb} / \mathrm{s}$ and comes in both multimode and single-mode flavours for short and long reach data transmission respectively. In the field of Accelerator Instrumentation at CERN, the VTRx together with the serialiser/deserialiser GBTx ASIC [4] has been adopted by the GBT-based Expandable Front-End (GEFE) [5] project, which is the standard radiation-tolerant digital front-end for the Beams Instrumentation (BI) group. This work develops next generation single-mode optical links for accelerator instrumentation based on the Versatile Link framework. It is desirable that the new optical datalink generation supports higher data rate than the first generation, in order to cope with the high data volume produced by the beam instrumentation. The new VTRx is intended to be mounted onto the next generation radiation-tolerant digital frontend for the BI group together with the low-power GBT (lpGBT) ASIC. This optical transceiver will be installed in the harsh radiation environment next to the beam pipe. The radiation field varies depending on the installation site along the accelerator. It has been estimated that in the expected lifetime ( 10 years) the radiation levels could reach a $10 \mathrm{kGy}$ total dose and a $5 \cdot 10^{14}$ $20 \mathrm{MeV}$ neutrons $/ \mathrm{cm}^{2}$ particle fluence.

The first part of the paper shows the static and dynamic characterization of a number of edge-emitting-laser (EEL) candidates to replace the part, which became obsolete, used in the first module generation. The devices were also tested for displacement damage and TID effects up to the maximum irradiation levels. The second part of the paper presents solutions to increase the uplink data rate into a single fiber to a maximum of $40 \mathrm{~Gb} / \mathrm{s}$.

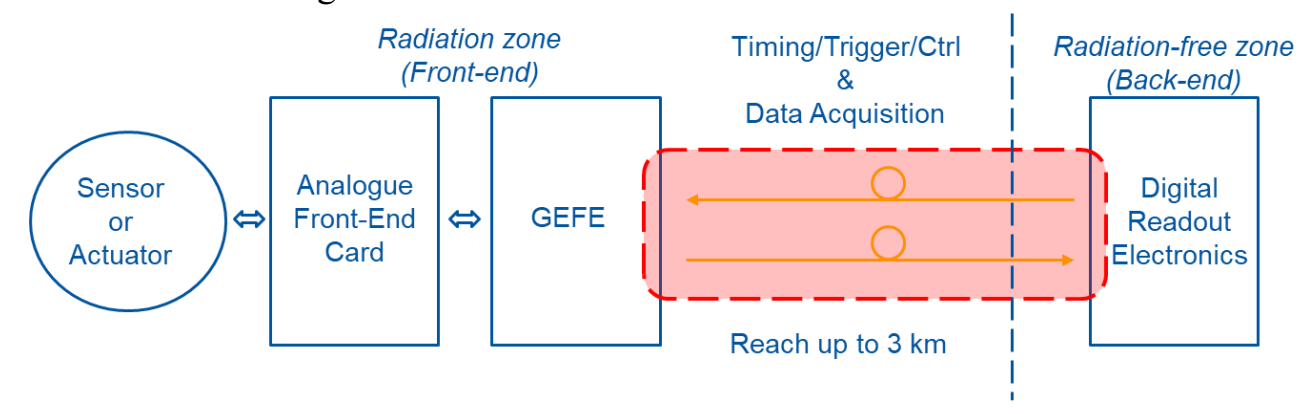

Figure 1 Block diagram of a sensing and control system for particle accelerators at CERN. Optical data links with length up to $3 \mathrm{~km}$ are used to transfer data between back-end and front-end electronics 


\section{Edge-Emitting Laser}

This section presents the selection process for the Laser diode. Ten commercial off-the-shelf (COTS) edge-emitting Lasers, operating at $1310 \mathrm{~nm}$ and compatible with the VTRx design, were procured and tested. The following paragraphs report on methods and results of the measurement campaign. Laser types are indicated with letters from A to L.

\subsection{Functional characterization}

Test vehicle for the optical eye diagram measurement was the single-mode VTRx, Figure 2 (Left), on which the DUT was mounted. Bias $\left(\mathrm{I}_{\text {bias }}\right)$ and modulation $\left(\mathrm{I}_{\mathrm{mod}}\right)$ currents were set in the Laser driver (GBLD) using I2C communication. Measurement conditions were: GBLD default current settings ( $\mathrm{I}_{\text {bias }}=40 \mathrm{~mA}$ and $\mathrm{I}_{\mathrm{mod}}=20 \mathrm{~mA}$ ) and input data pattern PRBS-7 at $4.8 \mathrm{~Gb} / \mathrm{s}$. From the eye diagram it is possible to extract parameters related to optical modulation amplitude, time jitter and frequency response. The radar plot in Figure 2 (Right) shows the eye diagram parameters for all the tested devices. For easier evaluation, the parameters are normalized to the Versatile Link specifications [6]. All the points with value higher than one (points falling outside the red area on the radar plot) meet the specifications.

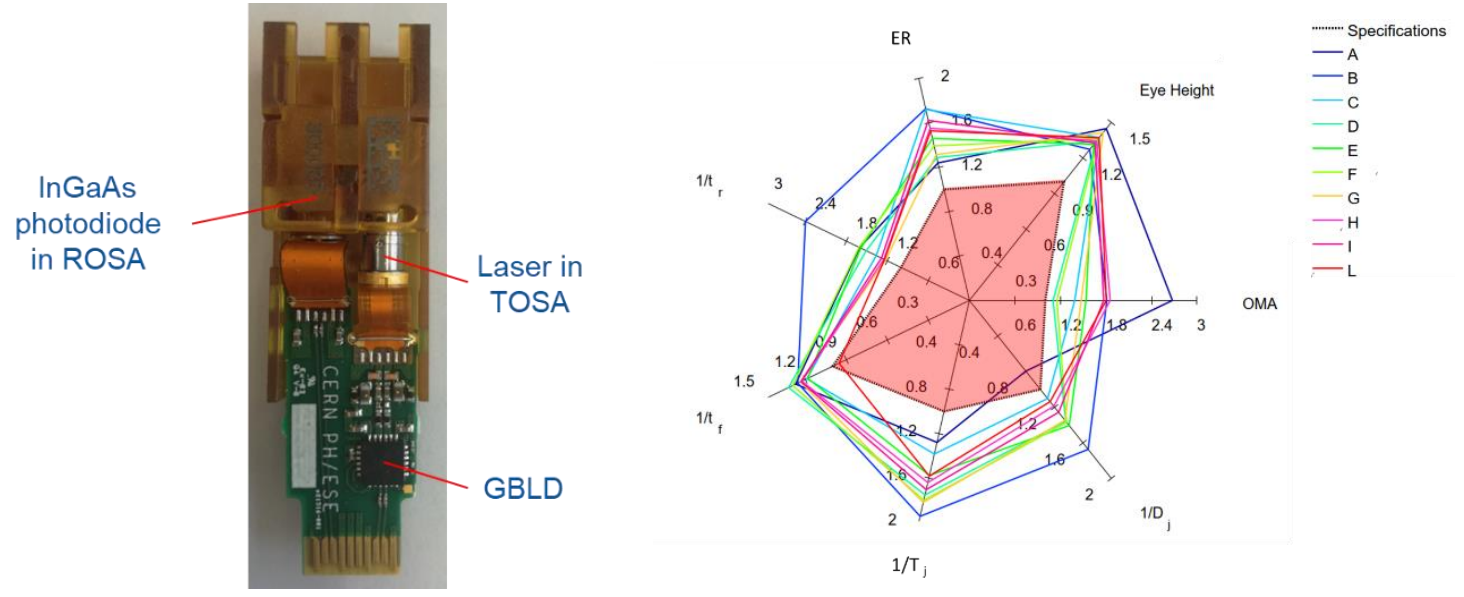

Figure 2 (Left) Picture of the first single-mode VTRx generation. (Right) Radar plot of the parameters extracted from the eye diagram for all the tested devices. The parameters were normalized with the Versatile Link specifications [6].

\subsection{Radiation tolerance}

Laser diodes are affected by atom displacement damage when exposed to particle fluence [7][8]. All the devices under test were irradiated with a $20 \mathrm{MeV}$ neutron beam with a total fluence of approximately $5 \cdot 10^{14} 20 \mathrm{MeV}$ neutrons $/ \mathrm{cm}^{2}$, which is with good approximation the maximum fluence expected in 10 years lifetime. The test was performed at the Cyclotron facilities in the Université Catholique de Louvain (UCL), Louvain-la-Neuve, BE.

L-I-V (light-current-voltage) curves were measured for all the devices at about twentyminute intervals throughout the irradiation that lasted about 48 hours. After the beam was switched off, the devices were left to recover for two weeks and during this time the measurement cycle continued.

Figure 3 shows the test results with the typical increase in Laser threshold current and decrease in slope efficiency. It is possible to identify a family of devices with moderate radiation 
induced damage (A, D, F, G, and $\mathrm{L}$ ) and another family with more severe radiation damage $(\mathrm{C}$, $\mathrm{H}$ and I). Note that the device $\mathrm{A}$ is the part mounted onto the first generation of single-mode VTRx, included in the test for comparison with the previous design.
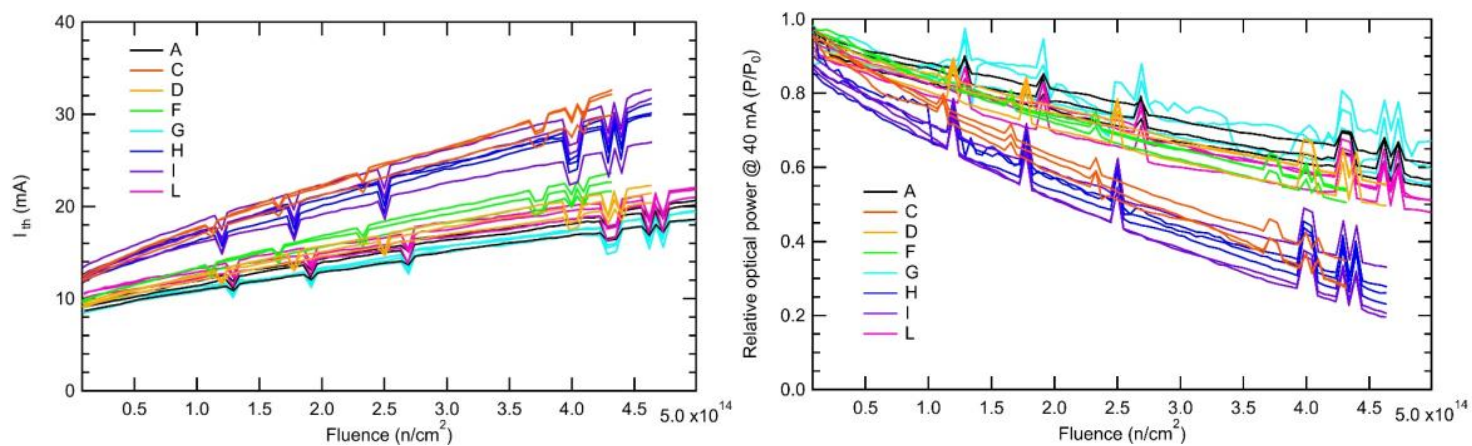

Figure 3 Threshold current and output optical power degradation during the neutron irradiation test for all the tested devices. The spikes visible on the plots are artefacts due to Laser temperature changes when the automated measurement system was rebooted. Device $G$ shows high radiation tolerance, comparable to device A that was used on the first VTRx generation.

\section{Uplink data rate}

The first VTRx generation is an optical transceiver with $4.8 \mathrm{~Gb} / \mathrm{s}$ symmetric uplink and downlink. Some Accelerator Instrumentation applications require uplink capacity up to $40 \mathrm{~Gb} / \mathrm{s}$ per fiber. We addressed this by increasing the lane rate from $4.8 \mathrm{~Gb} / \mathrm{s}$ up to $10.24 \mathrm{~Gb} / \mathrm{s}$ (compatible with the lpGBT) that was possible thanks to the high analog bandwidth of the selected COTS edge emitting laser and the modulation current pre-emphasis circuit implemented in the GBLD laser driver. Figure 4 (Left) shows the optical eye diagram of the VTRx operating at $10 \mathrm{~Gb} / \mathrm{s}$ when the pre-emphasis on the modulation current is activated. Figure 4 (Right) shows the bit error rate curves for a reference receiver when the optical input signal is generated by the VTRx operated at $5 \mathrm{~Gb} / \mathrm{s}$ and $10 \mathrm{~Gb} / \mathrm{s}$, and in both cases with and without pre-emphasis. The two curves at $5 \mathrm{~Gb} / \mathrm{s}$ overlap, while the receiver sensitivity when the VTRx is operated at $10 \mathrm{~Gb} / \mathrm{s}$ with default settings is of about $2 \mathrm{~dB}$ worse with respect to the case with pre-emphasis enabled. Based on these results, we conclude that the VTRx can be operated at $10 \mathrm{~Gb} / \mathrm{s}$, with a minimum impact on the link power budget, in applications that require high data rate uplink.
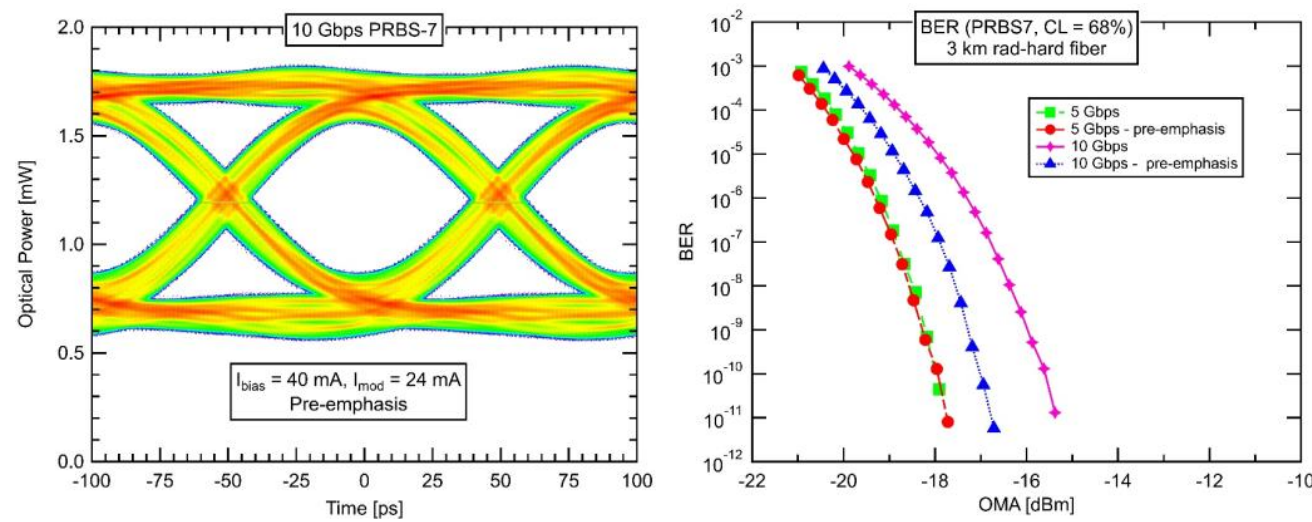

Figure 4 (Left) Eye diagram of the VTRx at $10 \mathrm{~Gb} / \mathrm{s}$ with pre-emphasis enabled on the modulation current. (Right) Comparison of bit error rate curves of a 'reference' receiver when the optical input signal is produced by a VTRx operated in 4 different conditions: $5 \mathrm{~Gb} / \mathrm{s}$ and $10 \mathrm{~Gb} / \mathrm{s}$ in both cases with and without pre-emphasis on the modulation current. 


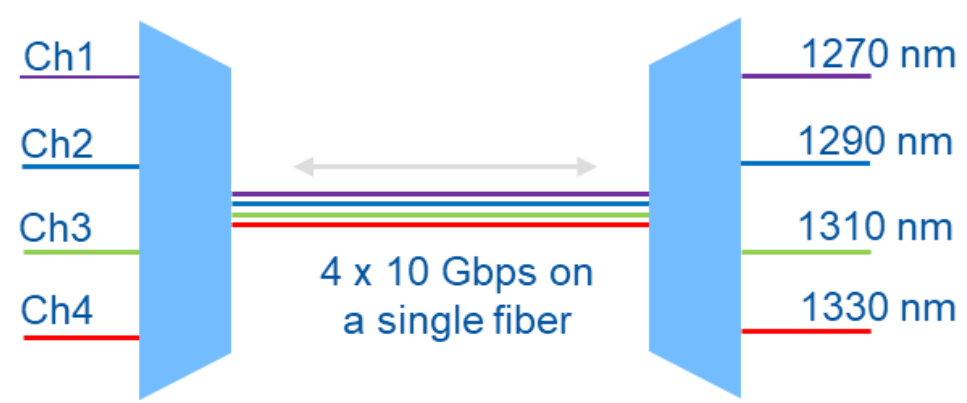

Figure 5 Coarse wavelength division multiplexing (CWDM) scheme: four channels (10 Gbps) multiplexed into a single-mode fiber.

For systems requiring data rate per fiber higher than $10 \mathrm{~Gb} / \mathrm{s}$, we plan to implement Coarse Wavelength Division Multiplexing (CWDM) that aggregates into the same fiber four $10 \mathrm{~Gb} / \mathrm{s}$ channels operating at wavelengths spaced $20 \mathrm{~nm}$ apart: $1270 \mathrm{~nm}, 1290 \mathrm{~nm}, 1310 \mathrm{~nm}$ and 1330 $\mathrm{nm}$, Figure 5.

\section{Summary}

During the CERN Long Shutdown LS3 and LS4 several sensing and control systems of the SPS and the LHC will be upgraded. Radiation-hard and high-speed optical data links are a fundamental part of such systems. This work focuses on the development of a new generation of single-mode optical transceivers based on the Versatile Link framework that supports lane rate of $10.24 \mathrm{~Gb} / \mathrm{s}$ in the uplink and capacity of $40 \mathrm{~Gb} / \mathrm{s}$ per fiber when a wavelength division multiplexing is used. Further work involves radiation tolerance validation of CWDM Laser diodes and optical multiplexers.

\section{References}

[1] F. Vasey et al., The Versatile Link common project: feasibility report, 2012 JINST 7 C01075

[2] G. Mazza et al., A 5Gb/s Radiation Tolerant Laser Driver in $130 \mathrm{~nm}$ CMOS technology, 2012 JINST $7 \mathrm{C} 01052$

[3] M. Menouni et al., The GBTIA, a 5 Gbit/s radiation-hard optical receiver for the SLHC upgrades, in Proc. TWEPP 2009 Topical Workshop on Electronics for Particle Physics, September, 21-25, 2009 Paris, France, CERN-2009-006

[4] P. Moreira et al., The GBT Project, in Proc. of TWEPP 2009 Topical Workshop on Electronics for Particle Physics, September, 21-25, 2009 Paris, France, CERN-2009-006

[5] M. Barros Marin et al., The Giga Bit Transceiver based Expandable Front-End (GEFE) - a new radiation tolerant acquisition system for beam instrumentation, 2016 JINST 11 C02062

[6] Versatile Link Specifications: https:/edms.cern.ch/nav/P:CERN-0000076379:V0/P:CERN$\underline{0000090391: \mathrm{V} 0 / \mathrm{TAB} 3}$

[7] J. Troska et al., Radiation damage studies of lasers and photodiodes for use in multi-Gb/s optical data links, IEEE Transactions on Nuclear Science, Vol. 58, No. 6, December 2011

[8] J. Troska et al., Laser and photodiode environmental evaluation for the Versatile Link project, 2013 $\underline{\text { JINST } 8 \text { C02053 }}$ 